\title{
Theonyme in der baskisch-keltischen Kontaktzone Aquitaniens
}

\author{
F.E.R.C.AN-Arbeitstreffen in Vitoria-Gasteiz am 26. September 2000
}

1. Die große Verbreitung der Kelten in weiten Gebieten Europas und des angrenzenden Asiens wird als Ausdehnungsprozeß verstanden, der während einer längeren Zeitspanne von einem kleineren Kern ausging, dessen geographische und zeitliche Bestimmung umstritten ist. Von diesem Ausdehnungsprozeß verlieren sich einige Bewegungen im Dunkel der Vorgeschichte - man kann nur unvollkommene Hypothesen wagen, die aus der Analyse archäologischer Daten hervorgehen -, während andere, neuere Bewegungen durch die klassischen Quellen registriert wurden, wie der Einfall in Rom oder der Raubzug nach Delphi. ${ }^{1}$

Das Panorama wird noch komplizierter, wenn wir bedenken, daß es innerhalb des Keltenbegriffs mehr als eine Art und Weise gibt, Kelte/keltisch zu sein, abhängig von kulturellen Phänomenen unterschiedlicher Epochen. Identifizierte man «Keltisch» bis zur Mitte des 20. Jahrhunderts fast vollständig mit «Gallisch» und mit der La Tène-Kultur, so führten die Entdeckung weiterer "Keltikai» einerseits, wie die keltiberische oder die lepontische, und die fehlende Verbindung zwischen goidelischer Sprache und einer materiellen La Tène-Kultur in Irland andererseits ${ }^{2}$, dazu, daß der Keltenbegriff auf Bereiche erweitert wurde, die man vorher vernachlässigt hatte.

Aus dieser Perspektive können wir uns vorstellen, daß die letzte Phase des Keltischen - das sprachlich, aber auch künstlerisch und hinsichtlich der materiellen Kultur und Religion als die Entwicklung des Gallischen im Kerngebiet der weiten keltischen Welt verstanden werden kann - in einigen Gebieten archaischere Entwicklungsstufen des Keltischen überlagerte; dies läßt sich im Falle Italiens dank der Überlieferung im Alpenraum und im Norden feststellen ${ }^{3}$, ist aber auch für weniger bekannte Gebiete denkbar. Wenn man

1 Vgl. aus sprachwissenschaftlicher Sicht Karl Horst ScHмIDT, The Celtic Problem. Ethnogenesis (location, date?), in: ZCP 45 (1992) 38-65; für prähistorische Fragen Javier de Hoz, The Celts of the Iberian Peninsula, in: ZCP 45 (1992) 1-37; zu den klassischen Texten, die sich auf Kelten beziehen, H. D. Rankin, The Celts through Classical Eyes, in: Miranda J. Green (ed.), The Celtic World. London - New York 1995. Eine rezente Neubewertung zum Thema der Völkerkontakte und -bewegungen in historischer Zeit aus archäologischer Sicht bei J.-J. CHARPY (ed.), L' Europe celtique du Ve. au IIIe. siècle avant J.-C. Contacts, échanges et mouvements de populations, Sceaux 1995.

2 Vgl. Gearóid Mac Eorn, The Celticity of Celtic Ireland, in: K. H. Schmidt (ed.), Geschichte und Kultur der Kelten, Heidelberg 1986, 161-174, wo der Gegensatz zwischen einem keltischsprachigen Irland einerseits und dem fast gänzlichen Fehlen materieller Reste der La Tène-Kultur andererseits hervorgehoben wird. Ausführlicher aber zum Grundsätzlichen: H. BIRKHAN, Kelten. Versuch einer Gesamtdarstellung ihrer Kultur, 3. korrigierte Auflage Wien 1999, 43-46.

3 Hinsichtlich des Materials vgl. die Artikel zu den Kelten Italiens in dem Sammelband The Celts, Milano 1991: Raffaele Carlo De Marinis, Golasecca Culture and its links with Celts beyond the Alps, 93-102, in dem auf die Ursprünge der ersten Phasen Mai- 
mit Interesse Brunaux' Beschreibungen und Deutungen der großen gallischen Heiligtümer im Nordwesten Frankreichs liest ${ }^{4}$, die man der „belgischen Expansion“ zuschreibt, fragt man sich: „Welchen Bevölkerungstyp überlagerte diese Expansion? Keltisch oder nicht keltisch?“

2. Ist die Überlagerung einer früheren Schicht durch irgendeine keltische Phase zu einer verhältnismäßig späten Epoche geschehen, so besteht für die besagte frühere Schicht die Möglichkeit, indirekt ans Licht zu kommen, durch historische Nachrichten in den klassischen Quellen oder durch sprachliche Zeugnisse in sekundärer Überlieferung, hauptsächlich aber in der Onomastik des betreffenden Gebietes (Toponymie bzw. Personennamengebung). Ein klares Beispiel liefert uns Südfrankreich, genauer gesagt die Narbonensis, deren kaiserzeitliche lateinische Epigraphik zwei gut differenzierte onomastische Korpora liefert, ein echt keltisch-gallisches entlang der Rhone und an ihrer Mündung (mit Elementen wie Excingus, Litu-, -maro- usw.) und ein anderes, das eher auf die Zone östlich der Rhone begrenzt ist (mit PNN wie Velianius, Pedanius, Ennius usw. $)^{5}$. Die kürzlich entdeckte griechische Bleiinschrift aus Pech Maho (Aude), in der Nähe von Narbonne, die in das erste Drittel des 5. Jhs. v. Chr. datiert wird, zeugt von der dortigen Anwesenheit von Völkerschaften, die weder Iberer noch Gallier waren (diese treten erst in der späteren iberischen Dokumentation auf), sondern sicherlich als die einheimische Bevölkerungsschicht der Gegend zu identifizieren sind ${ }^{6}$. Neben ganz klar iberischen Namen wie Basigerros, Nalbeadin oder Golobiur haben wir auch Personennamen wie Bleruas und Jauaruas, die der einheimischen, nicht-iberischen Bevölkerungsschicht entsprechen müssen.

Die sprachliche Zuschreibung dieser überlagerten Bevölkerungsschichten ist eine schwierige Aufgabe. Eine Unterscheidung zwischen indogermanisch und nichtindogermanisch dürfte theoretisch am wenigsten problematisch sein, sofern man über in dieser Hinsicht einigermaßen entscheidende Daten verfügt. Dies scheint mir zumindest im Süden der Iberischen Halbinsel der Fall zu sein, im turdetanischen Gebiet, das später, jedoch noch zur Zeit vor der römischen Eroberung, in seinem westlichen Teil einen keltischen $\mathrm{Zu}-$ wachs erhalten sollte, wenn auch F. Villar vor kurzem die ONN auf -uba, die man traditionell als vorindogermanisch auffaßte, als indogermanisch inter-

lands in der Golasecca-Kultur hingewiesen wird; Daniele VITALI, The Celts in Italy, 220235, über die materiellen und historischen Aspekte der durch die klassischen Quellen bekannten keltischen Völker. Zu den linguistischen Aspekten vgl. MichelLEJEUnE, Lepontica, Paris 1971, und Recueil des Inscriptions Gauloises (=RIG) II.1: Textes gallo-étrusques. Textes gallo-latins sur pierre, Paris 1988, bes. „Alphabet nord-étrusque et langue gauloise“, 3-8.

4 J.-L. Brunaux, Les religions gauloises. Rituels de la Gaule indépendante, Paris1996, $34 \mathrm{f}$.

5 Jürgen Untermann, Lengua gala y lengua ibérica en la Galia Narbonensis, in: Archivo de Prehistoria Levantina 12 (1969) 99-161; idem, Quelle langue parlait-on dans l' Hérault pendant l' Antiquité?, in: Revue Archéologique de Narbonnaise 25 (1992) 19-27.

${ }^{6}$ Der Text wurde von Michel Lejeune, Jean Poullloux und Yves Solier publiziert: Étrusque et ionien archaïques sur un plomb de Pech Maho (Aude), in: Revue Archéologique de Narbonnaise 21 (1988) 19-59; die einheimische Onomastik behandelt Joaquín Gorrochategui, Los Pirineos entre Galia e Hispania: las lenguas, in: Veleia 12 (1995) 187 und 191, sowie vor allem Javier de Hoz, Los negocios del señor Heronoios. Un documento mercantil jonio clásico temprano, del Sur de Francia, in: J. A. López FÉREz (ed.), Desde los poemas homéricos hasta la prosa griega del s. IV d. C., Madrid 1999, 61-90. 
pretiert hat, was eine chronologische „Revolution“ in der Auffassung der Indogermanisierung Hispaniens impliziert ${ }^{7}$.

Ist die überlagerte Schicht eine indogermanische, vermehren sich die Identifikationsprobleme ungemein; dies kann der Fall der in der Narbonensis beobachteten Schicht sein, die Untermann - als Erbe einer langen Tradition - mit dem Namen "Ligurisch" versah und in deren jüngster ethnischer Kristallisierung sich indogermanische Zuwächse mit einem vorindogermanischen Substrat summiert haben können. In dieser Hinsicht sind viele Gebiete des indogermanischen Teils der Iberischen Halbinsel sehr problematisch. $\mathrm{Da} ß \mathrm{im}$ Westen Lusitanisch gesprochen wurde, dessen Zuordnung für die Mehrheit der Sprachwissenschaftler nicht keltisch ist, ist uns lediglich durch drei erhaltene und zwei verlorene Inschriften bekannt ${ }^{8}$. Dennoch zeigen gerade einige dieser Inschriften Eigennamen, die als keltisch klassifizierbar sind; wir wissen, daß es in relativ jüngerer Zeit Bewegungen von Völkerschaften keltischen Ursprungs gab, die durch Lusitanien hindurch bis in die Gegend des Guayana kamen, und die - wie uns Plinius ${ }^{9}$ deutlich sagt - aus Keltiberien stammten. Da das Lusitanische eine uns praktisch unbekannte Sprache ist, kennen wir bis auf einige wenige Ausnahmen das Isoglossenbündel, das es vom Keltischen unterscheidet, nicht im einzelnen, so daß wir nicht wissen, wie wir die Fälle einordnen sollen, in denen die beiden Sprachen übereinstimmen; warum soll man z. B. denken, das Element trebo-, belegt in dem GN Trebopala oder auch in Trebarune, sei keltisch und nicht lusitanisch bzw. einer unabhängigen westlichen indogermanischen Sprache zugehörig? Sind wir so sicher, daß der Wurzelvokal kurz wie im Keltischen und nicht lang wie im Italischen (vgl. osk. tríbúm) ist? Es gibt keine keltischere Gottheit als Lugus, der

7 Für eine Darbietung des Materials und die Probleme, die sich aus den südlichen Daten ergeben, ist zu vergleichen: J. de Hoz, El desarrollo de la escritura y las lenguas de la zona meridional, in: $\mathrm{M}^{\mathrm{a}} \mathrm{E}$. AUBET (ed.), Tartessos y sus problemas. V Symposium internacional de prehistoria peninsular, Barcelona 1989, 523-587, und Tartesio, fenicio y céltico, 25 años después, in: Tartessos 25 años después. Actas del Congreso conmemorativo del V Symposium int. de prehistoria peninsular, Jerez de la Frontera 1995, 591607. Der Vorschlag von Francisco VILLAR, der in seiner umfassendsten und neuesten Version in Indoeuropeos y no indoeuropeos en la Hispania prerromana, Salamanca 2000, zu finden ist, stellt uns vor schwer lösbare historische Probleme, abgesehen von Fragen der Methode.

8 Zur sprachlichen Einordnung des Lusitanischen ist eine Mehrheit der Auffassung, es als nicht-keltisch westindogermanisch anzusehen, die auf Antonio Tovar, L'inscription de Cabeço das Fráguas et la langue des lusitaniens, in: Études Celtiques 11, (1964/ 67) 237-268, zurückgeht und mit der ich übereinstimme (Joaquín GoRROCHATEGUI, En torno a la clasificación del lusitano, in: Studia palaeohispanica. Actas del IV Coloquio sobre lenguas y culturas paleohispánicas = Veleia $2-3$, Vitoria 1987, 77-91), sowie eine andere, im wesentlichen von Jürgen UnTERManN, Lusitanisch, Keltiberisch, Keltisch, in: Studia Palaeohispanica = Veleia 2-3 (1987) 57-76, vertretene, die es als eine weitere keltische Sprache ansieht; auch A. L. Prosdocimi hat sich der keltistischen Hypothese angeschlossen, allerdings mit einem linguistischen Argument (Alter der heteroklitischen Flexion der lusitanischen $a$-Stämme, vgl. Aldo Luigi Prosdocimi, L’inscrizione gallica del Larzac e la flessione dei temi in -a, -i, -ja. Con un ,excursus' sulla morfologia del lusitano: acc. crougin, dat. crougeai, in: IF 94 (1989) 190-205), das sich philologisch nicht absichern läßt (vgl. Joaquín GoRRochateguI, La declinación céltica de los temas en - $a$ y los datos hispanos, in: Indogermanica et Caucasica. Festschrift K. H. Schmidt. (eds. R. Bielmeier \& R. Stempel), Berlin - New York 1994, 316-330).

9 Plinius III, 13: Quae autem regio a Baete ad fluuium Anam tendit extra praedicta, Baeturia appellatur, in duas diuisa partes totidemque gentes: Celticos...Turdulos...Celticos a Celtiberis ex Lusitania aduenisse manifestum est sacris, lingua, oppidorum uocabulis, quae cognominibus in Baeturia distinguntur. (Pline l'ancien. Histoire naturelle, livre III, texte établi, traduit et commenté par H. Zehnacker, Paris 1998). 
in allen keltischen Gebieten des Kontinents belegt ist, wie auch in der längsten keltiberischen Inschrift aus Peñalba de Villastar. Wir wissen, daß es in einigen Gegenden Galiziens Kelten gab, weil es uns Plinius sagt und auch wegen einiger ONN auf -brig->-bri- sowie wegen der ethnischen Identifikation einiger Völker wie im Falle der Supertamarici Celtici. In einigen Inschriften aus der Provinz Lugo - bedeutender, wenn auch nicht entscheidender Name (denn er läßt sich einfach aus lat. Lūcum erklären) - finden wir folgende Gottheit belegt: Lucoubu Arquien; Lucubo Arquinob, im Plural, was man als den Ausdruck der bekannten pankeltischen Gottheit ansah; mit einer zwar hyperkritischen Haltung hat Javier de Hoz jedoch einige Einwände gegen eine solche Identifikation vorgebracht, was die Schwierigkeiten zeigt, mit der wir uns in komplexen Gebieten bewegen, die sprachlich nicht einheitlich sind ${ }^{10}$.

3. Bevor ich auf das aquitanische Gebiet zu sprechen komme, möchte ich als letzten Punkt en passant einen weiteren methodologischen Zugriff auf das uns interessierende Problem zitieren, zumal er unmittelbar das Thema der gallischen Religion betrifft. Jean-Jacques Hatt dachte ${ }^{11}$, daß die gallische Religion, so wie sie in ihren gallo-römischen Denkmälern auf uns gekommen ist, eine synkretistische Religion war, allerdings nicht nur im Sinne einer Verbindung des keltischen mit dem römischen Element, sondern daß das, was man gewöhnlich als keltisch versteht, eigentlich ein komplexes Konglomerat religiöser Vorstellungen war, die man unterschiedlichen Phasen oder Schichten zuschreiben könnte: Einige Elemente gingen auf die vorherige Bevölkerung zurück, und andere, die wirklich keltisch waren, könnten sogar verschiedenen Phasen des Keltischen zuzuschreiben sein. Hatt unterscheidet bis zu fünf Schichten innerhalb der gallischen Religion: a) alte vorgeschichtliche Schicht, b) neolithische Periode, mit einer Göttin der „Mutter Erde“, c) ausgehende Bronzezeit, mit einiger Sicherheit mittels der geographischen Verteilung des einheimischen Marskultes wiederzugewinnen: „répartition des Mars indigènes, comparée à celle des premiers établissements celtiques en Gaule... Il s'agit de divinités principalement masculines, souvent associées à des parèdres féminines... Elles sont plurifonctionnelles, correspondent à un groupe humain socialement organisé, elles sont fortement implantées dans le sol, dans le milieu social. Ces divinités topiques, remontant à la révolution technologique et sociologique du Bronze Final, ont survécu avec leurs caractères dominants au cours des périodes suivantes, grâce au cloisonnement des civilisations et à la longue survivance, dans certaines régions, des traditions du Bronze Final. Ces dieux seront adorée à l'époque romaine sous le nom générique de Mars"; d) hybride Mischung aus der Hallstattzeit (Pferdegott, Herrschende Göttin, chthonische Gottheit - ausgedrückt durch die Schlange usw.) und e) gallische Religion der La Tène-Zeit, in der die Götter einen Namen haben: Taranis, Teutates, Esus usw.

Der Karte der Belege des Mars in Gallien kommt m. E. keine besondere Bedeutung zu, denn obwohl sie in den Pyrenäen und in der Narbonensis zahlreich sind - was mit unserer Information aus anderen Quellen übereinstimmen würde -, finden wir eine beträchtliche Anzahl von Widmungen an Mars

10 Javier de Hoz, Lingua e etnicidade na Galicia antiga, in: Gerardo Pereira Menaut (ed.), Galicia fai dous mil anos. O feito diferencial galego: I. Historia 1, Santiago de Compostela 1997, 101-140.

11 Jean-Jacques Hatr, Mythes et dieux de la Gaule, Paris 1989. 
Caturix unter den Helvetiern, an Mars Camulus unter den Remi und an Mars Segomo unter den Sequani, und zwar ohne daß man diese Kulte vorkeltischen Schichten zuschreiben könnte. Schließlich gibt es keine Zeugnisse eines Marskultes auf der ganzen südlichen, am Atlantik gelegenen Seite, ein allem Anschein nach spät keltisiertes Gebiet.

4. Viele direkte und auch indirekte Zeugnisse zeigen uns, daß Aquitanien, genauer gesagt das ursprüngliche Aquitanien oder „petite Aquitaine“ zwischen den Pyrenäen und der Garonne, ein vom Gallischen und Keltischen deutlich abgegrenztes Gebiet war.

4.1. Darüber gibt es zwei eindeutige klassische Zitate, eines zu Beginn des Bellum Gallicum:

Gallia est omnis diuisa in partes tres, quarum unam incolunt Belgae, aliam Aquitani, tertiam qui ipsorum lingua Celtae, nostra Galli appellantur. Hi omnes lingua, institutis, legibus inter se differunt.

Das andere verdanken wir dem Geographen Strabo:

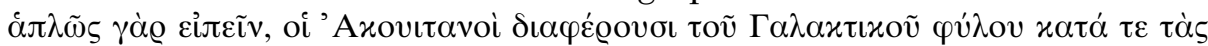

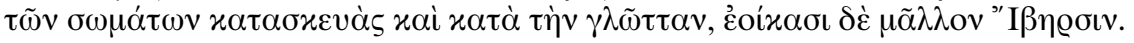

Wenn auch die mutmaßliche Ähnlichkeit der Aquitaner mit den Iberern bzw. besser gesagt mit den Einwohnern Iberiens - sehr viel Gesprächsstoff im Rahmen der basko-iberischen Hypothese geliefert hat, zeigen uns diese beiden Zeugnisse ausdrücklich, daß sich die Aquitaner deutlich von ihren nordöstlichen gallischen Nachbarn unterschieden ${ }^{12}$.

4.2. In Aquitanien ist kein einheimisches schriftliches Denkmal aufgefunden worden, das die von der Bevölkerung gesprochene Sprache direkt bezeugt. Es gibt lediglich einige Bruchstücke einer in Aubagnan (Landes, Frankreich) aufgefundenen Silberschale: Obwohl ihr fragmentarischer Charakter das Verständnis ungeheuer erschwert, s cheint es sich um einen iberischen Text zu handeln, der in diese Gegend als Luxusgut oder als Teil eines Schatzes gebracht worden sein könnte ${ }^{13}$. In diesem Sinne wird man auch den Fund des kleinen Schatzes von Barcus mit einer großen Menge hispanischen Geldes aus dem 1. Jh. v. Chr. verstehen.

4.3. Es bleibt uns die durch die kaiserzeitliche lateinische Epigraphik sekundär überlieferte Onomastik. Bereits 1877 bemerkte A. Luchaire ${ }^{14}$, daß viele pyrenäische und gaskognische Namen durch das Baskische eine gute Übersetzung erhielten, wenn auch andere identisch oder vergleichbar mit den Namen waren, die in anderen Gebieten Galliens belegt waren, wodurch sie als gallisch identifiziert werden durften. Es entstand dadurch ein Konflikt zwischen diesen beiden Extremen der Interpretation, der von jedem der späte-

12 Für eine Kommentierung dieser Texte und anderer Materialien, die sich auf die Aquitania Novempopulana beziehen, vgl. Joaquín GorRochateguI, Onomástica indígena de Aquitania, Bilbao 1984. Eine Vorstellung der aquitanischen Onomastik in deutscher Sprache ist: Joaquín Gorrochategui, Die vorrömische Onomastik Aquitaniens, in: Beiträge zur Namenforschung 22:4 (1987) 343-355.

13 Jürgen Untermann, Monumenta Linguarum Hispanicarum II, Wiesbaden 1980, 3812 (Inschrift B. 10); vgl. auch S. 45 der Einleitung. Die Untersuchung von J. C. HeBERT, Les deux phiales à inscriptions ibériques du tumulus no III de la lande „Mesplède“ à Vieille-Aubagnan (Landes), in: Bulletin de la Société de Borda-Dax 115 (No 417), 1990, 1-40, zeigt klar die mediterrane Herkunft der Schalen.

14 Achilles Luchaire, Les origines linguistiques de l' Aquitaine, in: Bul. Soc. des Sciences, Lettres et Arts de Pau (1876-77) 349-423. 
ren Gelehrten anders gelöst wurde, je nach seiner vorgefaßten Meinung und nach seinen Kenntnissen des einen oder des anderen Bereiches. Luchaire selbst ist in einer späteren Veröffentlichung aus dem Jahre 1879 viel keltophiler als in seinem ursprünglichen Werk ${ }^{15}$. Während Holder in seinem keltischen Namenschatz ${ }^{16}$ alles einschließt, was irgendeine Ähnlichkeit mit dem Keltischen hat, werten Hübner ${ }^{17}$ oder Schuchardt ${ }^{18}$ die Listen aquitanischer Namen für ihre iberistischen Vergleiche aus.

Das Problem ist schwierig, weil es zwar tatsächlich Namen gibt, die jeweils der einen oder der anderen Schicht angehören und manchmal leicht identifizierbar sind, in anderen Fällen aber die Tatsache, daß sie entweder keine charakteristischen Isoglossen zeigen oder keine Parallelen aufweisen, ihre Klassifizierung erschwert.

Diese Schwierigkeit wurde von K. H. Schmidt sehr richtig erkannt, der in seiner Dissertation über komponierte gallische Personennamen ${ }^{19}$ ein wichtiges Kapitel den hybriden Bildungen aus Kontaktzonen widmet: „Mischbildungen und Kontaktzonen":

„So können wir in vielen Fällen weder von der gallischen noch von der nichtgallischen Seite her das gallische Material klar herausschneiden. Keine Arbeit über das Gallische kann deshalb bei dieser Sprache selbst ansetzen. Immer muß erst „negative Arbeit“ (Weisgerber) in der Aussonderung alles Nichtgallischen geleistet werden. [...] Diese Namen verschiedenen Ursprungs haben sich dazu noch gegenseitig beeinflußt. Deshalb muß man neben der Scheidung und Trennung der den verschiedenen Sprachen angehörenden Namen auch die (durch 2 Sprachen hervorgerufenen) gegenseitigen Beeinflussungen besonders herausstellen. Hierbei können wir unterscheiden:

1. Als Mischkomposita im engeren Sinne kann man die hybriden Bildungen bezeichnen, bei denen sich ein gallisches (meist Hinter-) Glied mit einem fremden Bestandteil verbunden hat.

2. a) Der gallische Name begegnet uns durch das Medium eines fremden phonologischen Systems entstellt. [...]

3. Lehnübersetzungen von Kompositen oder einzelnen Kompositionsgliedern fremder Sprachen. [...]

4. Eine Art volksetymologischer Angleichung des fremden Wortes an ein gallisches hat stattgefunden.

5. Gallische Komposita sind mit fremden Suffixen versehen oder in eine fremde grammatische Struktur hineingeraten

6. Die Einzelglieder sind gallisch, aber die Art ihrer Zusammensetzung widerspricht den gallischen Kompositionsgesetzen." (S. 49-50)

Einen Teil dieser Kriterien wendet Schmidt ebenso bei der Erklärung einiger aquitanischer Namen an; so weisen z. B. Saleduna und Dunomagius semantische Probleme auf, will man sie als Zeugnisse des gallischen Elementes duno- ,Burg, Stadt‘ verstehen (in den eigentlichen gallischen Gebieten kommt dieses Element nicht in der Personennamengebung vor); daher „... könnte in Aquitanien ein an duno- anklingendes, fremdes Wort vorgelegen haben, wie der Dunoho-rix mit sicher ungall. 1. Gliede nahelegt. Dieses kann mit dem gall. duno- irgendwie verschmolzen sein“ (S. 200, wie Anm. 19).

15 Achilles Luchaire, Études sur les idiomes Pyrénéens de la région française, Paris 1879 (Faksimile-Ausgabe: Genève 1973).

16 Adolf HoLDER, Alt-celtischer Sprachschatz (3 Bände), Leipzig 1891-1913.

17 Emil HüBNER, Monumenta Linguae Ibericae, Berlin 1893.

18 Hugo SchuchaRd, Iberische Personennamen, in: Revue international d'études basques 3 (1909) 237-247.

19 Karl Horst Schmid, Die Komposition in gallischen Personennamen, in: ZCP 26 (1957) 31-301. 
5. Unter Beachtung dieser Vorsichtsmaßnahmen und mit Hilfe einer feineren und genaueren Analyse der Formen, sowohl in phonetischer und morphologischer wie auch in distributioneller Hinsicht, ist es gelungen, Unterschiede bei einigen Namen festzustellen. So war es etwa üblich, die aquitanischen Namen mit seni (wie Senius, Seniten-, Senixo-, Senipon- usw.) an die keltische Wurzel seno- ,alt‘ anzuschließen, weil die äußere Ähnlichkeit groß ist und es sich um ein im Keltischen gut belegtes Element handelt. Michelena ${ }^{20}$ war der erste, der sich darüber Rechenschaft ablegte, daß das aquitanische Wort doch systematisch auf - $i$ lautete, was es vom Gallischen entfernte; zur Erklärung gelangte er, als er die baskische Parallele sehi, sein ,puer, $\pi \alpha \tilde{\mathbf{I}} \varsigma^{\prime}$ in der doppelten Bedeutung von ,Junge“ und ,Knecht‘ heranzog, mit regelmäßigem Schwund von intervokalischem /n/ in einer Periode, die später als die der lateinischen Lehnwörter ist. Umgekehrt ist Sennacius, wegen der Gemination wie wegen des Suffixes, von dieser Gruppe zu trennen und läßt sich besser vom Gallischen her erklären.

Das aquitanische Wort für die Frau, Andere, und seine Ableitungen ( $A n$ derese, Anderexso, And(e)reco), identisch mit baskisch andere ,Frau, Dame“, wurde mit keltisch *andera ,Färse, junge Frau' in Verbindung gebracht. Die Keltologen (Vendryes, LEIA) ${ }^{21}$ sprechen von einem Substratwort, und die Baskologen, immer um eine Etymologie verlegen, akzeptieren die keltische Verbindung. Man muß sich aber klarmachen, daß erstens der semantische Wert in beiden Sprachen verschieden ist (bask. ,Frau, Großmutter': kelt. ,junge Frau') und daß zweitens das dreisilbige andere - an und für sich schon eine Ausnahme vom Morphemkanon - innerhalb des aquitanischen Korpus mit dem Frauennamen Ere- zu vergleichen ist: wir können eine Korrelation aufstellen $^{22}$ :

$\begin{array}{lll}\text { Frauennamen: } & \text { Ere- } & \text { Andere } \\ \text { Männernamen: } & \text { Dox-us } & \text { Andox(us) }\end{array}$

Der Name aquit. Dannadinnis (Gen.) erlaubt für sein erstes Element einen Vergleich mit gall. danno-, das keine sehr klare Bedeutung hat, aber in Namen von Würdenträgern oder Beamten wie argantodannos und in Personennamen wie Dannotalos vorkommt. Der zweite Bestandteil auf -adinn jedoch, mit klaren Parallelen in vielen iberischen Namen, läßt uns eine iberische Klassifikation vorziehen und daran denken, daß es sich ursprünglich um einen Namen handelt, dessen beide Elemente iberisch sind: Tanneg-adin, das an die aquitanische Phonetik angepaßt und vielleicht durch das gallische Element danno- beeinflußt wurde ${ }^{23}$.

Wenn baskische Parallelen fehlen oder nicht sehr präzise sind, kommen uns Zweifel. Es wurde schon der Fall des Frauennamens Saledunae (Dat.) erwähnt, in dem Schmidt einen „nicht-gallischen“ Gebrauch des Elementes

20 Luis Michelena, De onomástica aquitana, in: Pirineos 10 (1954) 409-458 [= idem, Lengua e Historia, Madrid 1985, 409-445]

21 Joseph Vendryes, Lexique étymologique de l'irlandais ancien, Lettre A, Paris 1959.

22 Eine interne Erklärung aller dieser Namen bei Joaquín GoRRochATEGUI und Joseba A. LAKARRA, Nuevas aportaciones a la reconstrucción del protovasco, in: Francisco VILLAR und José D'Encarnação (eds.), La Hispania prerromana. Actas del VI Coloquio sobre Lenguas y Culturas paleohispánicas, Salamanca 1995, 101-145.

${ }^{23}$ Die nominalen Elemente, die Teil der iberischen Onomastik sind, behandelt Jürgen UnTERMANN, Monumenta Linguarum Hispanicarum III: Die iberischen Inschriften aus Spanien, 1. Literaturverzeichnis, Einleitung, Indices. Wiesbaden 1990, bes. $\S 6$ und $\S 7$ (195-238). 
duno- sah. In diesem Falle ist vielleicht die Parallele anderer Frauennamen aus demselben Korpus besser, wie z. B. Aedunniae (Dat.) oder Edunxe, obwohl uns keine späteren baskischen Parallelen vorliegen.

Wie soll man Namen wie Orgot erklären? Unmittelbar fällt uns der Name des helvetischen Heerführers Orgeto-rix ein, dessen erstes Element, orgeto-, ein Nomen agentis zu einer in air. org(a)id, schlägt, verletzt' gut belegten Wurzel ist. Im aquitanischen Korpus gibt es jedoch andere Namen, mit denen Orgot in Beziehung steht, etwa Orcot[, Orcotarris (Gen.), Orgoanno, Orguarrae, die dem gallischen Bereich fernstehen.

Hat schließlich, im gleichen Sinne, der Name des Volks der Tarbelli etwas mit gall. tarbo- ,Stier' zu tun, wie es ohne Zweifel in dem Namen Tarbelsonios in einer gallischen Inschrift aus Vieux-Poitiers vorliegt, oder eher mit den aquitanischen Elementen tar- und -bel, die respektive in dem Ortsnamen Tarròs sowie häufig in Personennamen wie Harbel- vorkommen?

6. Unter Berücksichtigung der von Schmidt genannten Kriterien habe ich die Personennamen Aquitaniens folgendermaßen klassifiziert: a) echt aquitanische, b) gallische, c) gemischte. Die aquitanischen Namen sind auf dem gesamten aquitanischen Territorium zu finden, mit größerer Häufigkeit in den Pyrenäen; die gallischen sind entlang der Flüsse Garonne und Salat belegt und dringen weder in die Pyrenäen noch in die Zentral-Gascogne vor; die gemischten, zu denen nicht nur die hybriden, sondern auch die an die aquitanische Phonetik angeglichenen sowie die mit einheimischen Suffixen abgeleiteten zählen, sind stärker im Westen vertreten und schon in gaskognischem Gebiet belegt; sie zeichnen zu einem großen Teil den Weg für die Linie der größten Verbreitung der Suffixe auf -acum vor, die im westlichen Aquitanien nicht vorkommen ${ }^{24}$.

7. Was die Götternamen betrifft, so sind genaue Vergleichsmomente mit der einen oder der anderen Seite seltener. Jedenfalls liegen einige wenige klare Fälle keltischer Gottheiten vor:

\section{Minervae Belisamae.}

Carpento deo (drei Belege), vgl. gall. carbanto-.

Sehr wahrscheinlich keltisch ist Arpenino deo, wenn wir an *ar(e)penn(o)- ,Kopf" (eigentlich frühneuhochdeutsch für haupt ,Stirnseite des Feldes' und ,Ackermaß'; vgl. Birkhan, Kelten ${ }^{3}$, 1101) plus Suffix -ino-s denken, sowie ein verlorengegangener, als Exprcennio gelesener Göttername.

Eine Weihinschrift, die Hirschfeld ohne Zweifel als Montibus Numidis las, könnte dem Keltischen zugeschrieben werden, wenn nimidis zu lesen wäre, wie einige wollten, aber die Lesung müßte überprüft werden.

Ferner ist es möglich, das Theonym Belgoni deo mit der Wurzel *bhelgh,schwellen` zu verbinden, die dem Ethnikon Belgae ,die Zornigen` zugrunde liegt.

In den Götternamen Edelati und Horolati erkennen wir ein onomastisches Element gall. -lati, das z. B. in Segolatius vorliegt, aber es fehlt an gallischen Parallelen für die ersten Bestandteile.

Ein Theonym mit vielen Belegen, aber - im Gegensatz zu anderen - mit

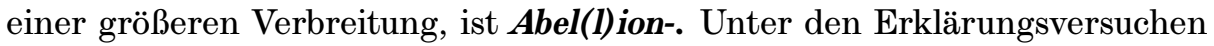
ist vielleicht jener der beste, der ihn mit ky. awel ,Wind, Sturm‘ (gr. $\alpha \dot{\alpha} \lambda \lambda \alpha \alpha$ )

${ }^{24}$ Um die Verteilung dieser Namentypen anhand von Karten nachzuvollziehen, vgl. J. Gorrochategui, wie Anm. 6, 206-211. 
verbindet; wir hätten dann eine Anpassung von kelt. /w/ an aquit. /b/ sowie die lateinische Parallele in der Inschrift von Auch (CIL 441): Ingenua Ventis $v s l m$.

Ein Göttername, der innerhalb des aquitanischen Korpus wegen seiner Form fremdartig wirkt, ist die in St-Béat verehrte Gottheit $\operatorname{Er}(\boldsymbol{r})$ iap(p)o: Er enthält ein /p/, das im aquitanischen System nicht verankert ist, und weist sonst nicht belegte Geminaten auf ${ }^{25}$. Es wurde vorgeschlagen, darin eine Aquitanisierung des lateinischen Gottes Priapus zu sehen.

Über die einheimischen Götternamen hinaus existieren weitere, nichtrömische, autochthone Gottheiten, die unter lateinischem Namen angerufen werden. $\mathrm{Zu}$ dieser Gruppe gehören Baumnamen wie Fago deo (man beachte das maskuline Genus, obwohl lat. fagus feminin ist) oder die eher seltenen Sexsarboribus und Sexsarbori (Sing.). Als aquitanische Korrelate sind zu nennen: Artahe, Artehe, Arte, die Gottheit der Steineiche (vgl. bask. arte ,Steineiche'), und Arixo ,Eiche“(vgl. bask. aritz ,Eiche'). Gelegentlich treffen wir sogar auf einen Synkretismus mit Mars: Marti Arixoni (CIL 366). Ebenfalls auf gallischem Gebiet belegt ist eine Widmung an die ,Eiche“ (vgl. Robori, Angoulême, $C I L 1112$ = ILA, Santons, 98), und in beiden Fällen handelt es sich um den lateinischen Ausdruck eines primitiven und autochthonen Kultes für die Naturkräfte.

8. Aquitanische Personen- und Götternamen kommen auch außerhalb der eigentlichen Grenzen Aquitaniens vor. Die umfangreichste Personennamengruppe stammt von den silbernen Votivtäfelchen, die im Rhein nahe bei Hagenbach gefunden wurden ${ }^{26}$. In Narbonne $(C I L 12,4316)$ ist eine Widmung an Herculi Ilunno Andose belegt; das erste „Epitheton“ (falls es sich hier nicht um das zweite Glied eines synkretistischen Götterformulars und damit um einen Götternamen handelt), Ilunno, ist sehr häufig im oberen Luchon-Tal, und das zweite, Andose, ist eine Variante von Andosso, das an zwei Stellen ebenfalls als Epitheton belegt ist: Bascei Andosso (nahe St-Béat) und Herculi Toli Andosso im Gers. Die weite Verbreitung des Namens spricht eher für eine allgemeine als für eine lokale oder toponymische Bedeutung. Verbinden wir ihn des weiteren mit dem überaus häufigen aquitanischen Anthroponym Andoxus/Andossus, so können wie den Bedeutungsansatz ,dominus' wagen, der gleichermaßen zu einer Gottheit (vgl. die lateinische Parallele Marti domino) wie als männliches Gegenstück zu dem Frauennamen Andere paßt, der, wie oben gesagt, ,Dame = domina' bedeutet.

25 Leo Weisgerber, Zum Namengut der Germani Cisrhenani, in: Rhenania GermanoCeltica, Bonn 1969, 275-296, machte auf eine bemerkenswerte Übereinstimmung in der cisrhenanischen und aquitanischen Onomastik aufmerksam, die in dem Vorkommen von Suffixen oder Endungen mit Doppelkonsonanten in drei- oder mehrsilbigen Wörtern besteht; für die mitteleuropäischen Fälle deutete er als mögliche Ursache ein vorindogermanisches Substrat an. Zu diesem Thema vgl. auch Helmut BIRKHAN, Zur keltischen Namenkunde (Besprechung von L. Weisgerber, Rhenania Germano-Celtica), in: Blätter für deutsche Landesgeschichte 108, 1972, 202-210.

${ }^{26}$ Diese Bleche wurden veröffentlicht von Helmut BERnHARDT, Heinz-Josef Engels, Renate Engels, Richard Petrovszky, Der römische Schatzfund von Hagenbach, Mainz 1990 und speziell R. EngELs, Zur Herkunft der Votivbleche aufgrund der Namen, 1419. Für eine Untersuchung der aquitanischen Onomastik dieser Bleche vgl. Joaquín Gorrochategui, wie Anm. 6, 212-215, und Idem, Las placas votivas de plata de origen aquitano halladas en Hagenbach (Renania-Palatinado, Alemania), in: Revue Aquitania [im Druck]. 
Dieser Vergleich zwischen Götterepitheta und Personennamen, der sich hier als fruchtbar erwiesen hat, kann allerdings nicht auf alle Fälle bloß aufgrund äußerer Ähnlichkeit ausgedehnt werden. So haben wir etwa in den Pyrenäen den Personennamen Cison und seine Ableitungen Cisonten, Cissonbonnis und Gisondonis, die seit Luchaire mit bask. gizon ,Mann“ in Verbindung gebracht werden (vgl. dessen lateinische Übersetzung in dem Titulla Homulli filia einer Inschrift aus St-Béat, CIL 40), während in der rezenten Edition der Inschriften von Santons eine dekorierte Bronzetafel mit einer Widmung an Mercurio Cisonio publiziert ist. Dazu lautet der Kommentar von Maurin ${ }^{27}$ :

„Ex-voto dédié à une divinité celtique, Cisonius ou Cissonius, que l'on trouve généralement invoquée sous le nom de Mercure Cissonius. Les documents sur cette divinité (une douzaine d'inscriptions au total) sont échelonnés sur le Rhin moyen, région où devait se trouver le berceau du culte, de Strasbourg à Cologne, et secondairement à Besançon, Metz et Trèves. Certaines de ces inscriptions montrent que des influences orientales se sont exercées au IIe. s. sur Mercure Cissonius (CIL 13, 5273 = ILS 4598), comme de son côté le manifeste l'iconographie de notre petite plaque. Témoignage complètement isolé de ce culte dans l'Ouest de la Gaule, cet ex-voto avait vraisemblablement été apporté à Saintes des régions rhénanes par un marchand ou un soldat" (S. 234).

Auch wenn wir Dokumente aus dem Rheinland gesehen haben, die ihren Ursprung in den aquitanischen Pyrenäen haben, scheint es doch klüger zu sein, sich in diesem Falle nicht von der äußerlichen Ähnlichkeit hinreißen zu lassen, sondern jede einzelne dieser Formen innerhalb ihres geographischen und linguistischen Umfeldes zu erklären.

Vorgelegt von w. M. GERHARD Dobesch in der Sitzung am 21. Juni 2002.

27 Louis MAurin, Inscriptions latines d'Aquitaine (ILA): Santons, Bordeaux 1994. 